\title{
An Estimate on Convergence of Approximation by Iterations of a Solution to a Quasi-Variational Inequality and Some Consequences on Continuous Dependence and $G$-Convergence $\left(^{*}\right)$.
}

\author{
Marco Biroli (Milano)
}

Summary. - Si dà una stima per l'approssimazione mediante iterazione della soluzione di una disequazione quasi-variazionale e se ne deducono risullati circa la dipendenza continua della soluzione dall'ostacolo e dal termine noto e circa la G-convergenza.

\section{1. - Introduction and results.}

Let be $\Omega \subset \boldsymbol{R}^{N}$ a bounded open set with smooth boundary $\partial \Omega$ and $a_{i j} \in L^{\infty}(\Omega)$, $i, j=1, \ldots, N$, such that

$$
\sum_{i j=1}^{N} a_{i j}(x) \xi_{i} \xi_{j} \geqslant \alpha|\xi|^{2} \quad \text { a.e. in } \Omega
$$

$\forall \xi \in \boldsymbol{R}^{N}$; we suppose $0 \in \Omega$.

We indicate, by $H_{\Gamma}^{1}(\Omega)$ the subspace of $H^{1}(\Omega)$

$$
H_{\Gamma}^{1}(\Omega)=\left\{\left.u \in H^{1}(\Omega) \quad u\right|_{\Gamma}=0\right\}
$$

where $\Gamma \subset \partial \Omega$ is closed and regular (for the hypothesis on regularity of $\Gamma$ cf. [16]); we define $A: H_{\Gamma}^{1}(\Omega) \rightarrow\left(H_{\Gamma}^{1}(\Omega)\right)^{*}\left(\left(H_{\Gamma}^{1}(\Omega)\right)^{*}\right.$ is the dual of $\left.H_{\Gamma}^{1}(\Omega)\right)$ by

$$
\langle A u, v\rangle=\int_{\Omega} \sum_{i j=1}^{n} a_{i j}(x) u_{x_{i}}(x) v_{x_{j}}(x) d x+\lambda \int_{\Omega} u(x) v(x) d x
$$

(where $\lambda>0$ if $\Gamma \neq \partial \Omega, \lambda \geqslant 0$ if $\Gamma=\partial \Omega$ ) and $M: \mathfrak{L}^{\infty}(\Omega) \rightarrow \mathfrak{L}^{\infty}(\Omega)$ by

$$
M_{\varphi}(\mathrm{T})=\operatorname{Inf}_{\substack{\xi \geqslant 0 \\ x+\xi \in \Omega}}(\varphi(x+\xi)+K(x, \xi))
$$

$\forall \varphi \in \mathfrak{L}^{\infty}(\Omega)$, where $K(x, \xi) \in \mathfrak{L}^{\infty}\left(\Omega \times \boldsymbol{R}_{+}^{n}\right) \quad\left(\boldsymbol{R}_{+}^{n}=\left\{\xi \in \boldsymbol{R}^{n}, \xi \geqslant 0\right\}\right) K(x, \xi) \geqslant K_{0} \geqslant 0$.

(*) Entrata in Redazione il 29 marzo 1978. 
Let be $f \in \mathfrak{L}^{r}(\Omega), r>N$, with $f(x) \geqslant 0$ a.e. in $\Omega$; we consider the problem

$$
\left\{\begin{array}{ccc}
\langle A u, v-u\rangle \geqslant\langle f, v-u\rangle, & \\
\forall v \in H_{\Gamma}^{1}(\Omega) \quad v(x) \leqslant M u(x) & \text { a.e. in } \Omega, \\
u \in H_{\Gamma}^{1}(\Omega) & u(x) \leqslant M u(x) & \text { a.e. in } \Omega .
\end{array}\right.
$$

The problem (1.4) has been introduced by A. Bensoussan, J. L. Lions [1], [2], in relation to some problems in stochastic control; A. Bensoussan, M. Goursat, J. L. LIons [4], have shown the following existence result:

TH. 1. - Let be $u^{0}$ the solution to the problem

$$
A u^{0}=f
$$

and $u^{n}$ defined by

$$
\left\{\begin{array}{lll}
\left\langle A u^{n}, v-u^{n}\right\rangle \geqslant\left\langle f, v-u^{n}\right\rangle, & \\
\forall v \in H_{\Gamma}^{1}(\Omega) ; & v(x) \leqslant M u^{n-1}(x) & \text { a.e. in } \Omega \\
u^{n} \in H_{\Gamma}^{1}(\Omega) ; & u^{n}(x) \leqslant M u^{n-1}(x) & \text { a.e. in } \Omega
\end{array}\right.
$$

We have

$$
u_{n} \downarrow u \quad \text { in } \mathfrak{L}^{2}(\Omega)
$$

where $u$ is the maximum solution of the problem (1.4).

Th. Laestch has shown the following uniqueness result.

TH. 2. - Let be $K_{0}>0$; the solution to the problem (1.4) is unique.

In the framework of Th. 1,2 some results on continuous dependence can be obtained from Th. 4 [4]; these results have, however, some monotonicity hypothesis, which reduce their applicability.

In the framewock of Th. 1,2 some results on $G$-convergence for problems of type (1.4) can be obtained from [6].

These results are valid in the following hypothesis: let be $u_{0}$ a subsolution (cf. [20]) of the limit problem we define $u_{n}$ by

$$
\left\{\begin{array}{lll}
\left\langle A u_{n}, v-u_{n}\right\rangle>\left\langle t, v-u_{n}\right\rangle & \\
\forall v \in H_{\Gamma}^{1}(\Omega) ; \quad v(x) \leqslant M u_{n-1}(x) & \text { a.e. in } \Omega \\
u_{n} \in H_{\Gamma}^{1}(\Omega) ; & u_{n}(x) \leqslant M u_{n-1}(x) & \text { a.e. in } \Omega
\end{array}\right.
$$

the $u_{n} \uparrow u$, where $u$ is the solution of the limit problem. 
This hypothesis is difficult to verify and is related to the problem of the regularity of a solution of (1.4).

We specify, however, that the operators $M$ considered in [4], [6] are more general than the operator given by (1.3).

The aim of this paper is to obtain a more careful result on convergence of $u^{n}$ to $u$ than this of Th. 1 and to deduce from it some results on continuous dependence and $G$-convergence, which improve those of [4], [6].

We obtain:

TH. 3. - Let be $K_{0}>0$; we have

$$
0 \leqslant u^{n}-u \leqslant K \theta_{0}^{n}, \quad 0<\theta_{0}<1
$$

where $K, \theta_{0}$ are constants dependent only on $K_{0}$ and $\operatorname{Sup} u^{\circ}(x)$.

From Th. 3 we have a result shown by other methods by C. Trotanieldo [22]:

CoRoLl. 1. - Let be $K_{0}>0, K(x, \xi)=K(\xi) \in C\left(\boldsymbol{R}_{+}^{N}\right)$; the solution to the problem (1.4) is in $O(\Omega)$.

From Th. 3 we can deduce the following result on continuous dependence:

TH. 4. - Let be $\left\{f_{\alpha}\right\}\left\{K_{\alpha}\right\}$ two sequences such that

$$
\begin{gathered}
\lim _{\alpha \rightarrow+\infty} f_{\alpha}=f \quad \text { in } \mathfrak{L}^{\gamma}(\Omega), \quad r>N, \quad f_{\alpha}(x) \geqslant 0 \quad \text { a.e. in } \Omega \\
\lim _{\alpha \rightarrow+\infty} K_{\alpha}(x ; \xi)=K(x, \xi) \quad \text { in } \mathfrak{L}^{\infty}\left(\Omega \times \boldsymbol{R}_{\xi}^{N}\right), \\
K_{\alpha}(x ; \xi) \geqslant K_{0}>0 \quad \text { a.e. in } \Omega \times \boldsymbol{R}_{+}^{N}
\end{gathered}
$$

and $u_{\alpha}$ the solution to the problem

$$
\left\{\begin{array}{l}
\left\langle A u_{\alpha}, v-u_{\alpha}\right\rangle \geqslant\left\langle f, v-u_{\alpha}\right\rangle, \\
\forall v \in H_{\Gamma}^{1}(\Omega), \quad v(x) \leqslant M_{\alpha} u_{\alpha}(x) \quad \text { a.e. in } \Omega, \\
u_{\alpha} \in H_{\Gamma}^{1^{\prime}}(\Omega), \quad u_{\alpha}(x) \leqslant M_{\alpha} u_{\alpha}(x) \quad \text { a.e. in } \Omega .
\end{array}\right.
$$

Where

$$
M_{\alpha} \varphi(x)=\operatorname{Inf}_{\substack{x+\xi \in \Omega \\ \xi \geqslant 0}}\left(\varphi(x+\xi)+K_{\alpha}(x, \xi)\right)
$$

We have

$$
\lim _{\alpha \rightarrow+\infty} u_{\alpha}=u \quad \text { in } \mathfrak{L}^{\infty}(\Omega)
$$

where $u$ is the solution of (1.4). 
Let be now $a_{i i}^{p} \in \mathcal{L}^{\infty}(\Omega), i, j=1, \ldots, N$, such that

$$
\beta|\xi|^{2} \geqslant \sum_{i j=1}^{N} \alpha_{i j}^{p}(x) \xi_{i} \xi_{j} \geqslant \alpha|\xi|^{2} \quad \text { a.e. in } \Omega
$$

We define $A^{p}: H_{\Gamma}^{1}(\Omega) \rightarrow\left(H_{\Gamma}^{1}(\Omega)\right)^{*}$ by

$$
\left\langle A^{y} u, v\right\rangle=\int_{\Omega} \sum_{i j=1}^{n} a_{i j}^{p}(x) u_{x_{i}}(x) v_{x_{j}}(x) d x+\lambda \int_{\Omega} u(x) v(x) d x
$$

We denote by $u_{\text {s }}$ the solution to the problem

$$
\left\{\begin{array}{l}
\left\langle A^{p} u_{p}, v-u_{p}\right\rangle \geqslant\left\langle t, v-u_{p}\right\rangle, \\
\forall v \in H_{\Gamma}^{1}(\Omega) \quad v(x) \leqslant M u_{p}(x) \quad \text { a.e. in } \Omega, \\
u_{p} \in H_{\Gamma}^{1}(\Omega) \quad u_{p}(x) \leqslant M u_{p}(x) \quad \text { a.e. in } \Omega .
\end{array}\right.
$$

We suppose that $A^{p} G$-converges to $A$ (for the definition of $G$-convergence [8], [9], $[21])$.

Tн. 5. - If $K_{0}>0, K(x ; \xi)=K(\xi) \in C\left(\boldsymbol{R}_{+}^{N}\right), M: C(\bar{\Omega}) \rightarrow C(\bar{\Omega})$; we have

$$
\lim _{p \rightarrow+\infty} u_{p}=u \quad \text { in } C(\bar{\Omega})
$$

where $u$ is the solution of (1.4).

Remr. 1. - The result of Th. 5 improves a previous result of A. Bensoussan, J. L. Lrons [3], in which the $A$ suppose

$$
\lim _{y \rightarrow+\infty} a_{i j}^{p}=a_{i j} \quad \text { in } \mathfrak{L}^{\infty}(\Omega) \quad i, j=1, \ldots, N
$$

In the case of homogeneisation with $f$ also non positive and $K(x, \xi)=K_{0}>0$ we have a more precise estimate.

Let be $b_{i j}(x) i, j=1, \ldots, N$ in $C^{1}\left(\boldsymbol{R}^{N}\right)$ periodic of period $P=\prod_{i=1}^{N}\left[0, y_{i}\right]$ such that

$$
\beta|\xi|^{2} \geqslant \sum_{i j=1}^{n} b_{i j}(x) \xi_{i} \xi_{j} \geqslant \alpha|\xi|^{2}
$$

in $\boldsymbol{R}^{N} \forall \xi \in \boldsymbol{R}^{N}$.

We choose

and

$$
a_{i j}^{p}(x)=b_{i j}(p x)
$$

$$
a_{i j}(x)=q_{i j}
$$


where $q_{i j}$ are the constant coefficients of the homogeneised operator [5], [9], [13], [21].

In this case we have:

TH. 6. - Let be $f$ also non positive, $K(x, \xi)=K_{0}>0, \Gamma=\partial \Omega$

$$
g(x)=\operatorname{Inf}\left(\operatorname{Inf}_{\substack{x+\xi \in \Omega \\ \xi \geqslant 0}} f(x+\xi), 0\right)
$$

We suppose $g(x) \in \mathfrak{L}^{r}(\Omega)$ and $\underline{u}(x)>-K_{0}$ in $\Omega$ where $\underline{u}(x)$ is the solution to the problem

$$
A \underline{u}=g \text {. }
$$

We have

$$
\left\|u_{p}-u\right\| \mathbb{C}_{\infty} \leqslant C p^{\gamma}, \quad p \geqslant \bar{p}_{y}
$$

where

$$
\gamma=-\frac{\bar{\alpha}}{2(N-2+3 \bar{\alpha})}(1-\delta), \quad \forall \delta>0
$$

$(\bar{\alpha}$ is a possible Hölder coefficient in the De Giorgi-Nash theorem relative to $\alpha, \beta, \Omega, f$ ) and $\bar{p}_{\gamma}$ is an integer dependent on $\delta$.

In the $\S 2$ we show the Th. 3 and the Coroll. 1 ; in the $\S 3$ we give a proof of Th. 4 by the result of Th. 3 and the result of continuous dependence for variational inequalities [17], III, Th. 1.4; in the $\S 4$ we show the Th. 5 by Th. 3 and some preliminary lemmas on $G$-convergence for variational inequalities; in the $\S 5$ we give a proof of Th. 6 by Th. 3, the estimate on $G$-convergence for variational inequalities [7], and a result of J. L. Joly, U. Mosco, G. Trotanterlo [10], [15], on the regularity of solution to quasi-variational inequalities.

REM. 2. - A result analogous to Th. 3 for the problem in stochastic control related to (1.4) has been given by J. L. MENALDr [14]; unfortunately the equivalence between the two type of problems and approximations ask a regularity, which generally we are not able to show.

Rer. 3. - The Th. 3, 4, 5 can be extended to operators of the type

$$
\langle A u, v\rangle=\int_{\Omega}\left\{\sum_{i=1}^{N}\left(a_{i j}(x) u_{x_{i}}(x)+b_{j}(x) u(x)\right) v_{x_{j}}(x) \sum_{i=1}^{N}\left(d_{i}(x) u_{x_{i}}(x)+c(x) u(x)\right) v(x)\right\} d x
$$

$u, v \in H_{I^{\prime}}^{1}(\Omega)$ where

$$
\begin{gathered}
\langle A u, u\rangle \geqslant \alpha\|u\|_{H_{T}^{1}}^{2} \quad \forall u \in H_{\Gamma}^{\mathrm{1}}(\Omega), \alpha>0 \\
a_{i j}, b_{j} d_{i}, c \in \mathcal{L}^{\infty}(\Omega), \quad c(x) \geqslant 0 \quad \text { a.e. in } \Omega .
\end{gathered}
$$


The Th. 3, 4 can be also extended to the case $\Omega$ unbounded if (1.5) is valid, and $K(x, \xi) \rightarrow+\infty$ for $|\xi| \rightarrow+\infty$ uniformly in $x$, and to the parabolic case if we consider the maximum solutions.

\section{2. - Proof of Th. 3 .}

Consider the problem

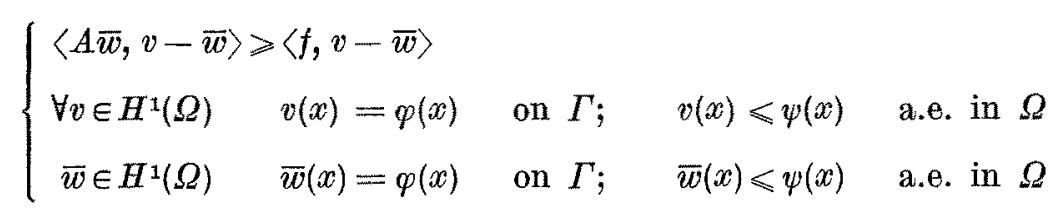

where $\varphi \in H^{\frac{1}{2}}(\partial \Omega), y \in \mathfrak{L}^{\infty}(\Omega)$ such that there is

$$
v \in H^{x}(\Omega), \quad v(x)=\varphi(x) \quad \text { on } \Gamma \quad \text { and } \quad v(x) \leqslant \psi(x) \quad \text { a.e. in } \Omega
$$

Let be $\bar{w}$ the solution of the problem (2.1); we indicate $\bar{w}=S(\psi, f, \varphi)$.

The Th. 3 is shown if we show

$$
u^{n}+Q \leqslant \frac{\theta_{0}^{-n}+O}{\theta_{0}^{-n}}(u+Q) \quad \text { a.e. in } \Omega
$$

where $0<\theta_{0}<1$ and $C, Q>0$ are suitable constants.

We show (2.2) by induction.

From [4] we have $u(x) \geqslant 0$ a.e, in $\Omega$; let be $P=\operatorname{Sup} u^{0}(x)<+\infty$; if $C Q \geqslant P$, we have (2.2) for $n=0$.

We suppose now (2.2) for $n-1$ and we show (2.2) for $n$.

We have

$$
u^{n-1}+Q \leqslant \frac{\theta_{0}^{-n+1}+C}{\theta_{0}^{-n}}(u+Q) \quad \text { a.e. in } \Omega
$$

Let be $w^{n}=u^{n}+Q, w^{n-1}=u^{n-1}+Q, w=u+Q$.

We have

$$
w^{n}=S\left(M w^{n-1}, f+\lambda Q, Q\right)
$$

We observe that (2.2) is equivalent to

$$
\frac{\theta_{0}^{-n}}{\theta_{0}^{-n}+C} w^{n} \leqslant w
$$


We have

$$
\begin{gathered}
\frac{\theta_{0}^{-n}}{\theta_{0}^{-n}+C} w^{n}=S\left(\frac{\theta_{0}^{-n}}{\theta_{0}^{-n}+O} M w^{n-1}, \quad \frac{\theta_{0}^{-n}}{\theta_{0}^{-n}+O}(f+\lambda Q), \frac{\theta_{0}^{-n}}{\theta_{n}^{0-}+C} Q\right) \\
w=S(w,(f+\lambda Q), Q) .
\end{gathered}
$$

From [17] Th. 1.4, we have

$$
\frac{\theta_{0}^{-n}}{\theta_{0}^{-n}+C} w^{n} \leqslant S\left(\frac{\theta_{0}^{-n}}{\theta_{0}^{-n}+C} M w^{n-1}, \quad f+\lambda Q, Q\right) .
$$

$\Delta s$ in [12], pg. 165, we have

$$
M\left(\alpha w^{n-1}\right) \geqslant \frac{\theta_{0}^{-n}}{\theta_{0}^{-n}+C} M w^{n-1} \quad(\alpha \geqslant 0)
$$

if

$$
\frac{1-\theta_{0}^{-n} /\left(\theta_{0}^{-n}+C\right)}{\theta_{0}^{-n} /\left(\theta_{0}^{-n}+C\right)-\alpha} \geqslant \frac{P+Q}{K_{0}}=\bar{P}
$$

then

$$
\alpha=\max \left(\frac{\bar{P} \theta_{0}^{-n}-C}{\bar{P}\left(\theta_{0}^{-n}+C\right)}, 0\right)
$$

From (2.7), (2.8), (2.9) we have

$$
\frac{\theta_{0}^{-n}}{\bar{\theta}_{0}^{-n}+C} w^{n} \leqslant S\left(M\left(\alpha w^{n-1}\right), \quad f+\lambda Q, Q\right)
$$

then from (2.3)

$$
\frac{\theta_{0}^{-n}}{\theta_{0}^{-n}+C} w^{n} \leqslant S\left(M\left(\alpha \frac{\theta_{0}^{-n+1}+C}{\theta_{0}^{-n+1}} w\right), \quad f+\lambda Q, Q\right) .
$$

From (2.6) we have $(2.5)$ if

$$
\alpha \frac{\theta_{0}^{-n+1}+C}{\theta_{0}^{-n+1}} \leqslant 1 .
$$

We have (2.12) if $\alpha=0$; if $\alpha \neq 0$ we can write (2.12) as

$$
\begin{gathered}
\frac{\bar{P} \theta_{0}^{-n}-C}{\bar{P}\left(\theta_{0}^{-n}+C\right)} \frac{\theta_{0}^{-n+1}+C}{\theta_{0}^{-n+1}} \leqslant 1 \\
\bar{P} \theta_{0}^{-2 n+1}+\bar{P} C \theta_{0}^{-n}-C \theta_{0}^{-n+1}-C^{2} \leqslant \bar{P} \theta_{0}^{-2 n+1}+\bar{P} C \theta_{0}^{-n+1} \\
\bar{P} \theta_{0}^{-n}-(\bar{P}+1) \theta_{0}^{-n+1}-C^{2} \leqslant 0
\end{gathered}
$$


then we can choose

$$
\theta_{0}=\frac{\bar{P}}{\bar{P}+1}=\frac{P+Q}{P+Q+K_{0}}
$$

and we have (2.12) and (2.5).

The result is shown.

We give now the proof of Coroll. 1 .

LEMMA 1. - We consider $u=S(\psi, f, 0)$, where $f \in \mathfrak{L}^{\infty}(\Omega)$ and $\psi \in C(\bar{\Omega}), \psi \geqslant 0$ on $\Gamma$; we have $u \in C(\bar{\Omega})$.

From [15] we have the result if $A \psi \in \mathfrak{L}^{p}(\Omega), p>N$.

We can suppose $\varphi=0$ in $\Gamma$ we have a sequence $\left\{\psi_{n}\right\}$ such that

$$
\left\|A \psi_{n}\right\| \mathbb{L}_{\infty} \leqslant O \quad \lim _{n \rightarrow+\infty} \psi_{n}=w \quad \text { in } C(\bar{\Omega})
$$

then from [17] Th. 1.4; we have

$$
\lim _{n \rightarrow+\infty} S\left(\psi_{n}, f, 0\right)=S(\psi, f, 0) \quad \text { in } \mathfrak{L}^{\infty}(\Omega)
$$

then $u \in C(\bar{\Omega})$.

From [18] we have $u^{0} \in C^{\alpha}(\bar{\Omega})$ with $0<\alpha<1$; then from the lemma 1 and [14], [12] we have $u^{n} \in C(\Omega)$.

From Th. 3 we have

$$
\lim _{n \rightarrow+\infty} u^{n}=u \quad \text { in } \mathfrak{L}^{\infty}(\Omega)
$$

then $u \in C(\bar{\Omega})$.

REM. 4. - From the proof of Th. 3 we have that we can choose in the result

$$
\theta_{0}=\frac{\bar{P}}{\bar{P}+K_{0}}+\delta \quad \forall \delta>0 .
$$

In our proof we can not choose $\delta=0$, why for $\delta \rightarrow 0$ we have $Q \rightarrow 0$ and then $C \rightarrow+\infty$.

\section{3. - Proof of Th. 4 .}

From Th. 3 the Th. 4 is shown if we show that

$$
\lim _{\alpha \rightarrow+\infty} u_{\alpha}^{n}=u^{n} \quad \text { in } \mathfrak{L}^{\infty}(\Omega), \quad \forall n .
$$

We show (3.1) by induction. 
For $n=0$ we have

$$
A u_{\alpha}^{0}=f_{\alpha}, \quad A u^{0}=f
$$

then from [18], [19] we have

$$
\lim _{\alpha \rightarrow+\infty} u_{\alpha}^{0}=u^{0} \quad \text { in } \mathcal{L}^{\infty}(\Omega)
$$

We suppose now the result to be valid for $n-1$ and we show the result for $n$.

The function $u_{\alpha}^{n}$ is the solution of the problem

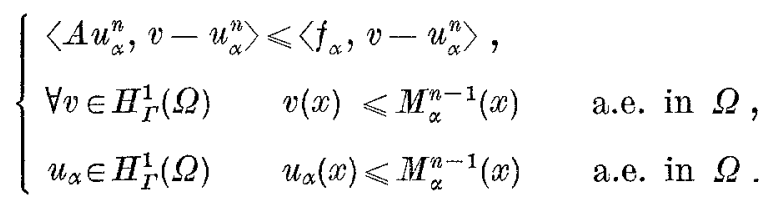

We indicate $w_{\alpha}=u_{\alpha}^{n}-A^{-1} f_{\alpha}$; then $w_{\alpha}$ is the solution to the problem

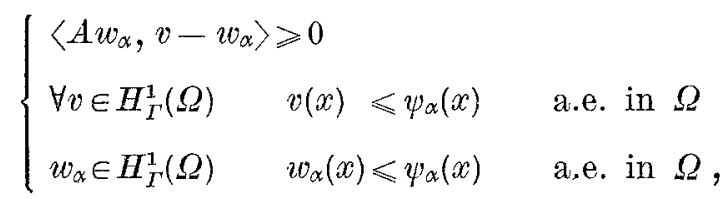

where

$$
\psi_{\alpha}(x)=M u_{\alpha}^{n-1}(x)-A^{-1} f_{\alpha}(x)
$$

Being

$$
\lim _{\alpha \rightarrow+\infty} f_{\alpha}=f \quad \text { in } \mathcal{L}^{r}(\Omega)
$$

we have [18], [19],

$$
\lim _{\alpha \rightarrow+\infty} A^{-1} f_{\alpha}=A^{-1} f \quad \text { in } C(\bar{\Omega})
$$

Being the result valid for $n-1$, we have

$$
\lim _{x \rightarrow+\infty} M u_{\alpha}^{n-1}=M u^{n-1} \quad \text { in } \mathfrak{L}^{\infty}(\Omega)
$$

then

$$
\lim _{\alpha \rightarrow+\infty}\left(M u_{\alpha}^{n-1}-A^{-1} f_{\alpha}\right)=\left(M u^{n-1}-A^{-1} f\right) \quad \text { inz } \mathcal{L}^{\infty}(\Omega)
$$

then from [17] Th. 1.4

$$
\lim _{\alpha \rightarrow+\infty} w_{\alpha}=w \quad \text { in } \mathcal{L}^{\infty}(\Omega)
$$


where $w$ is the solution to the problem

$$
\left\{\begin{array}{l}
\langle A w, v-w\rangle \geqslant 0 \\
\forall v \in H_{\Gamma}^{1}(\Omega) \quad v(x) \leqslant \psi(x) \\
w \in H_{\Gamma}^{1}(\Omega) \quad w(x) \leqslant \psi(x)
\end{array}\right.
$$

where

We have

$$
\psi(x)=M u^{n-1}(x)-A^{-1} f(x) .
$$

$$
w(x)=u^{n}(x)-A^{-1} f(x)
$$

then from (3.4), (3.7) we have

$$
\lim _{\alpha \rightarrow+\infty} u_{\alpha}^{n}=u^{n} \quad \text { in } \mathfrak{L}^{\infty}(\Omega)
$$

REM. 5. - The result of Th. 3 seem to be the first result on continuous dependence for solutions of quasi-variational inequalities without hypothesis on monotonicity for the sequences $\left\{f_{\alpha}\right\}\left\{K_{\alpha}\right\}$.

\section{4. - Proof of Th. 5 .}

We show at first the following lemma on $G$-convergence for variational inequalities:

LEMMa 2. - We consider the following problems

$$
\begin{aligned}
& \left\{\begin{array}{lll}
\left\langle A^{p} w_{p}, v-w_{p}\right\rangle \geqslant\left\langle f, v-w_{p}\right\rangle & \\
\forall v \in H_{\Gamma}^{1}(\Omega) & v(x) \leqslant \psi_{p}(x) & \text { a.e. in } \Omega \\
w_{p} \in H_{\Gamma}^{\mathrm{1}}(\Omega) & w_{p}(x) \leqslant \psi_{p}(x) & \text { a.e. in } \Omega
\end{array}\right.
\end{aligned}
$$

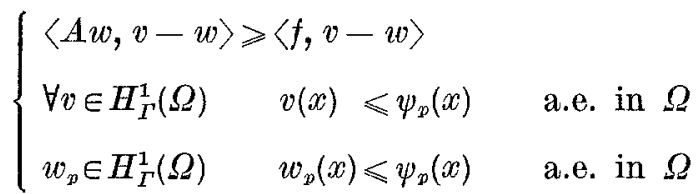

where $\psi_{p}, \psi \in C(\bar{\Omega})$

then

$$
\lim _{p \rightarrow+\infty} \psi_{p}=\psi \quad \text { in } C(\bar{\Omega})
$$

$$
\lim _{p \rightarrow+\infty} w_{p}=w \quad \text { in } C(\bar{\Omega})
$$

where $w_{p}(w)$ is the solution to $\left(4.1_{p}\right),((4.1))$.

We suppose at first $\left\|A^{p} \psi_{p}\right\|_{\mathfrak{L}^{\infty}} \leqslant$ Cst., $\|A \psi\|_{\mathfrak{L}^{\infty}} \leqslant$ Cst. 
From [16] we have

$$
\lim _{p \rightarrow+\infty} w_{p}=w \quad \text { in } \mathcal{L}^{2}(\Omega)
$$

and fron [15] Th.

$$
\left\|A^{n} w_{p}\right\|_{\mathfrak{L}} \leqslant \text { Cst. }
$$

then from [18] we have $\left\|w_{p}\right\|_{C^{\alpha}} \leqslant$ Cst., $0<\alpha<1$, and

$$
\lim _{p \rightarrow+\infty} w_{p}=w \quad \text { in } C(\bar{\Omega})
$$

We suppose now $\psi_{p}=\psi \in W^{1, p}(\Omega), p>N$, we can alse suppose $\left.\psi\right|_{r}=0$.

We prolongate $\psi$ to a function in $W^{1, p}\left(\boldsymbol{R}^{N}\right)$ then from the lemma of [9] we have a sequence $\left\{\psi_{p_{n}}\right\}\left\{\psi_{n}\right\}$ such that $\psi_{p_{n}},\left.\psi_{n}\right|_{\Gamma}=0$ and

$$
\left\{\begin{array}{l}
\left\|A^{p} \psi_{p}\right\| \mathbb{L}^{\infty},\left.\quad\left\|A \psi_{n}\right\|\right|_{L^{\infty} \leqslant C n^{\frac{1}{2}}} \\
\left\|\psi_{p n}-\psi\right\|_{L^{\infty}}, \quad\left\|\psi_{n}-\psi\right\| \|_{\mathcal{C}^{\infty} \leqslant C n^{-\frac{1}{2}}} \\
\lim _{p \rightarrow+\infty} \psi_{p n}=\psi_{n} \quad \text { in } C(\bar{\Omega}) \quad \forall n .
\end{array}\right.
$$

We consider now the problems

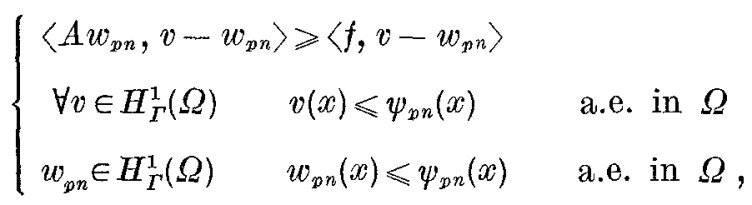

$$
\begin{aligned}
& \left\{\begin{array}{lll}
\left\langle A w_{n}, v-w_{n}\right\rangle \geqslant\left\langle f, v-w_{n}\right\rangle & \\
\forall v \in H_{\Gamma}^{1}(\Omega) \quad v(x) \leqslant \psi_{n}(x) & \text { a.e. in } \Omega \\
w_{n} \in H_{\Gamma}^{1}(\Omega) & w_{n}(x) \leqslant \psi_{n}(x) & \text { a.e, in } \Omega .
\end{array}\right.
\end{aligned}
$$

From the first part of the proof we have

$$
\lim _{p \rightarrow+\infty} w_{p n}=w_{n} \quad \text { in } C(\bar{\Omega}) \quad \forall n
$$

From (4.2) and Lemma 1.4 of [9] we have

$$
\left\{\begin{array}{l}
\left\|w_{m n}-w_{p}\right\|_{L^{\infty}} \leqslant C n^{\frac{1}{2}} \\
\left\|w_{n}-w\right\|_{L^{\infty}} \leqslant C n^{\frac{1}{2}}
\end{array}\right.
$$

7 - Annali di Malematica 
then

$$
\lim _{p \rightarrow+\infty} w_{p}=w \quad \text { in } C(\bar{\Omega})
$$

We suppose now $\psi_{p}, \psi \in W^{1, p}(\Omega), \psi_{p} \neq \psi$.

We indicate by $\bar{w}_{p}$ the solution to the problem

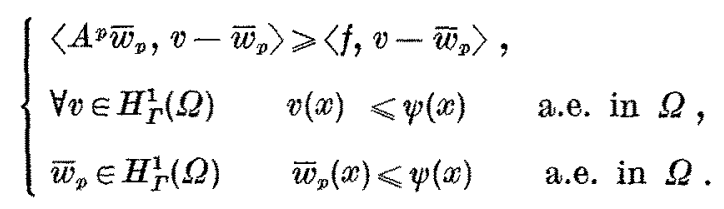

From (4.6) we have

$$
\lim _{p \rightarrow+\infty} \bar{w}_{p}=w \quad \text { in } C(\bar{\Omega})
$$

and from Th. $1.4[17]$

then

$$
\left\|w_{p}-\bar{w}_{p}\right\|_{\mathfrak{L}} \leqslant\left\|\psi_{p}-\psi\right\|_{\mathfrak{C}^{\infty}}
$$

$$
\lim _{p \rightarrow+\infty} w_{p}=w \quad \text { in } O(\bar{\Omega}) .
$$

We consider now the general case $\psi_{p}, \psi \in C(\bar{\Omega})$.

Being

$$
\lim _{p \rightarrow+\infty} \psi_{\bar{x}}=\psi \quad \text { in } O(\bar{\Omega})
$$

we have two sequences $\left\{\psi_{p k}\right\}\left\{\psi_{k}\right\}$ such that

$$
\begin{array}{ll}
\lim _{k \rightarrow+\infty} \psi_{p_{k}}=\psi_{p} & \text { in } C(\bar{\Omega}) \text { uniformly in } p . \\
\lim _{k \rightarrow+\infty} \psi_{k}=\psi & \text { in } C(\bar{\Omega}) \\
\lim _{p \rightarrow+\infty} \psi_{p k}=\psi_{k} & \text { in } O(\bar{\Omega}) .
\end{array}
$$

We indicate now by $w_{p t i}\left(w_{k}\right)$ the solution to the problem

$$
\begin{aligned}
& \left\{\begin{array}{lll}
\left\langle A^{n} w_{p k}, v-w_{p k}\right\rangle \geqslant\left\langle f, v-w_{p k}\right\rangle & \\
\forall v \in H_{\Gamma}^{1}(\Omega) & v(x) \leqslant \psi_{p k}(x) & \text { a.e. in } \Omega \\
w_{p k} \in H_{\Gamma}^{1}(\Omega) & w_{p k}(x) \leqslant \psi_{p k}(x) & \text { a.e. in } \Omega,
\end{array}\right.
\end{aligned}
$$

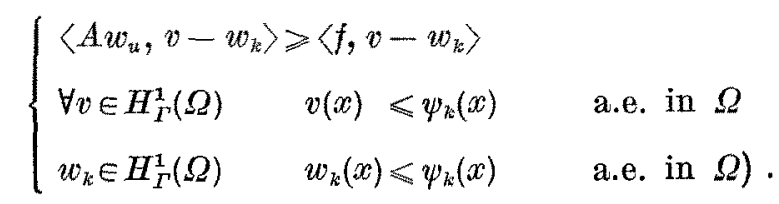


From (4.10) and Th. 1.4 [17] we have

$$
\lim _{k \rightarrow+\infty} w_{p \bar{k}}=w_{p} \quad \text { in } C(\bar{\Omega}) \text { uniformly in } p
$$

From (4.11), (4.12) and Th. 1.4 [17] we have also

$$
\begin{array}{ll}
\lim _{k \rightarrow+\infty} w_{k}=w & \text { in } C(\bar{\Omega}) \\
\lim _{k \rightarrow+\infty} w_{p k}=w_{k} & \text { in } C(\bar{\Omega}) .
\end{array}
$$

From (4.14) and (4.15) we have

$$
\lim _{p \rightarrow+\infty} w_{p}=w \quad \text { in } C(\bar{\Omega})
$$

We give now the proof of Th. 5 .

From [9], [21] we have

$$
\lim _{p \rightarrow+\infty} u_{p}^{0}=u^{0} \quad \text { in } \mathfrak{L}^{r}(\Omega)
$$

where

$$
A^{p} u_{p}^{0}=f
$$

From [18] we have $\left\|u_{p}^{0}\right\|_{0^{\alpha}} \leqslant$ Cst., $0<x<1$, then

$$
\lim _{p \rightarrow+\infty} \cdot u_{x}^{0}=u^{0} \quad \text { in } C(\bar{\Omega}) .
$$

We show now that

$$
\lim _{p \rightarrow+\infty} u_{p}^{n}=u^{n} \quad \text { in } C(\bar{\Omega})
$$

where $u_{p}^{n}$ is the solution to the problem.

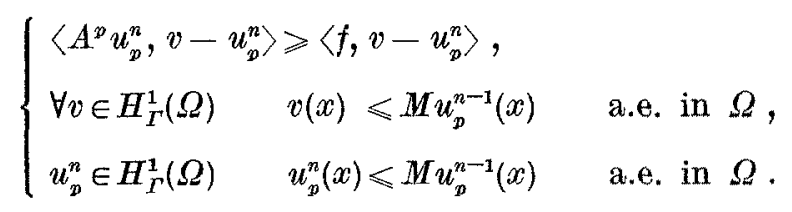

From (4.18) we have the result for $n=0$.

We suppose now the result for $n-1$ and we show the result for $n$.

Being

$$
\lim _{n \rightarrow+\infty} u_{p}^{n-1}=u^{n-1} \quad \text { in } C(\bar{\Omega})
$$


we have

$$
\lim _{p \rightarrow+\infty} M u_{p}^{n-1}=M u^{n-1} \quad \text { in } O(\bar{\Omega})
$$

then from Th. 1.4 [17]

$$
\lim _{y \rightarrow+\infty} u_{p}^{n}=u^{n} \quad \text { in } O(\bar{\Omega}) .
$$

From the Th. 3 we have also

$$
\left\{\begin{array}{l}
0 \leqslant u_{p}^{n}-u_{p} \leqslant K \theta_{0}^{n} \\
0 \leqslant u^{n}-u \leqslant K \theta_{0}^{n}
\end{array}\right.
$$

where the constant $K$ don ${ }^{s}$ depend on $p, n$.

From (4.23), (4.24) we have

$$
\lim _{p \rightarrow+\infty} u_{p}=u \quad \text { in } O(\bar{\Omega}) .
$$

\section{5. - Proof of Th. 6.}

Let be $B: H_{0}^{1}(\Omega) \rightarrow H^{-1}(\Omega)$ an elliptic operator with constant coefficients; we have [10], [15]:

Lemma 3, - Let be $f \in \mathfrak{L}^{r}(\Omega), r>n / 2$

$$
g(x)=\operatorname{Inf}\left(\operatorname{Inf}_{\xi \geqslant 0} \tilde{f}(x+\xi), 0\right) \in \mathcal{L}^{r}(\Omega)
$$

where $\tilde{f}$ is the prolongate of $f$ to $\boldsymbol{R}^{N}$ by 0 .

Let be $u$ the solution to the problem

$$
B \underline{u}=g .
$$

We suppose $\underline{u}(x) \geqslant-K_{0}$ a.e. in $\Omega$.

We consider the problem.

$$
\left\{\begin{array}{l}
\langle B u, v-u\rangle \geqslant\langle f, v-u\rangle, \\
\forall v \in H_{0}^{1}(\Omega) \quad v(x) \leqslant M u(x) \quad \text { a.e. in } \Omega, \\
u \in H_{0}^{1}(\Omega) \quad u(x) \leqslant M u(x) \quad \text { a.e. in } \Omega .
\end{array}\right.
$$

The problem (5.1) has a unique solution $u \in W^{2, p}(\Omega)$ and if we construct $u^{n}$ as in 
Th. 1 we have:

$$
\left\|B u^{n}\right\|_{\mathcal{L}^{r}} \leqslant \operatorname{Max}\left(\|f\|_{\mathcal{C}^{r}},\|g\|_{\mathfrak{C}^{r}}\right)
$$

LEMMA 4. - The result of Th. 3 is again valid for the problem

$$
\left\{\begin{array}{lll}
\langle A u, v-u\rangle \geqslant\langle f, v-u\rangle & \\
\forall v \in H_{0}^{1}(\Omega) \quad v(x) \leqslant M u(x) & \text { a.e. in } \Omega \\
u \in H_{0}^{1}(\Omega) \quad u(x) \leqslant M u(x) & \text { a.e. in } \Omega
\end{array}\right.
$$

(where $A$ is as in Th. 3 and f as in the Lemma 3) if $u(x)>-K_{0}$ in $\Omega$.

We observe that $\underline{u}(x)$ is continuous being $g \in \mathfrak{L}^{p}(\Omega)$, then we have

$$
\min _{x \in \bar{\Omega}} u(x)>-K_{0}
$$

Let be $w=u-\underline{u}, w$ is the solution to the problem

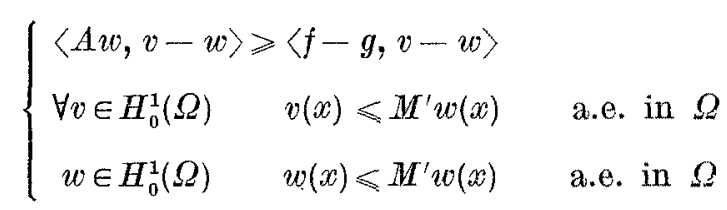

where

$$
M^{\prime} \varphi(x)=K_{0}-\bar{u}(x)+\operatorname{Inf}_{\substack{x+\xi \in \Omega \\ \xi \geqslant 0}}(\varphi(x+\xi)+\bar{u}(x+\xi))
$$

Let be now

$$
K(x ; \xi)=K_{0}-\underline{u}(x)+\underline{u}(x+\xi) .
$$

From (5.3) we have

$$
K(x ; \xi) \geq \delta>0
$$

From (5.5) we have

$$
M^{\prime} \varphi(x)=\operatorname{Inf}_{\substack{x+\xi \in \Omega \\ \xi \xi 0}}(\varphi(x+\xi)+K(x ; \xi))
$$

Finally we observe that $f-g \geqslant 0$.

Then the Th. 3 is valid for the problem (5.4) and we have

$$
0 \leqslant w^{n}-w \leqslant K \theta_{0}^{n}
$$


where $w^{0}$ is the solution to the problem

$$
A w^{0}=f-g
$$

and $w^{n}$ is the solution to the problem

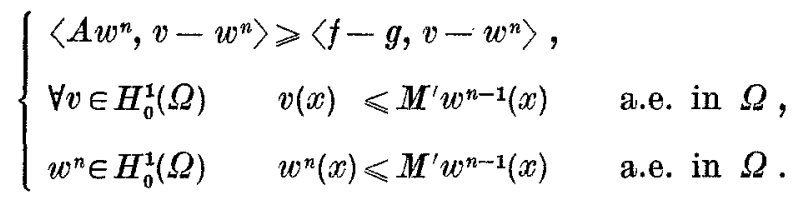

From (5.5), (5.8), (5.9) we have

$$
w^{n}=u^{n}-u, \quad \forall n
$$

then from (5.7)

$$
0 \leqslant u^{n}-u \leqslant K \theta_{0}^{n}
$$

LEMMA 5. - Let be $u \in H_{0}^{1, \infty}(\Omega)$ we have $M u \in H^{1, \infty}(\Omega)$ with

$$
\left.M u\right|_{\Gamma} \leqslant 0, \quad\|M u\|_{1, \infty} \leqslant\|u\|_{1, \infty} .
$$

Let be $u \in H_{0}^{1, \infty}(\Omega)$, then $M u \in C(\bar{\Omega})[14](12)$.

Let be $x, x+h e_{i} \in \Omega$, where $e_{i}$ is the unit vector in the direction of the $x_{i}, \xi \geqslant 0$.

We can suppose $h \geqslant 0$, the proof for $h \leqslant 0$ is analogous.

If $x+\xi$ and $x+h e_{i}+\xi$ are in $\bar{\Omega}$ we have:

$$
\left|u\left(x+h e_{i}+\xi\right)-u(x+\xi)\right| \leqslant C|h|
$$

where $C=\|u\|_{1, \infty}$.

If $x+h e_{i}+\xi \notin \Omega$, let be $\bar{h}=\theta h, 0<\theta \leqslant 1$, the supremum of the $\eta$ such that the segment $\left[x+\xi, x+\eta e_{i}+\xi[\right.$ is in $\Omega$.

We $x+\bar{h} e_{i}+\xi \in \partial \Omega$ then $u\left(x+\bar{h} e_{i}+\xi\right)=0$. We have

$$
|u(x+\xi)|=\left|u(x+\xi)-u\left(x+\bar{h} e_{i}+\xi\right)\right| \leqslant C|\bar{h}| \leqslant C|h| .
$$

Being $x+h \in \Omega$ there is $\xi^{\prime} \geqslant 0$ such that $x+h+\xi^{\prime} \in \partial \Omega$ then

$$
\left|u\left(x+h e_{i}+\xi^{\prime}\right)-u(x+\xi)\right| \leqslant C|h| .
$$

We can show analogously (5.11) in the case $x+\xi \notin \Omega, x+h e_{i}+\xi \in \Omega$.

If $x+\xi, x+h e_{i}+\xi \in \partial \Omega$ we have

$$
\left|u\left(x+h e_{i}+\xi\right)-u(x+\xi)\right|=0 .
$$


Then for $x, x+h e_{i} \in \Omega, \xi \geqslant 0, x+\xi \in \Omega$ there is a $\xi^{\prime} \geqslant 0$ such that

$$
\left|u\left(x+h e_{i}+\xi^{\prime}\right)-u(x+\xi)\right| \leqslant C|h| .
$$

The result of (5.13) is valid for $h \geqslant 0$ or $h \leqslant 0$, then

then

$$
\left|M u\left(x+h e_{i}\right)-M u(x)\right| \leqslant C|h|
$$

$$
\|M u\|_{1, \infty} \leqslant\|u\|_{1 ; \infty}
$$

Being now $\left.u\right|_{r}=0$ we have for all $x \in \Gamma$

$$
M u(x) \leqslant 0 \text {. }
$$

From [7], [8] we have

LEMMa 5. - Let be $\psi \in W^{1, r}(\Omega), r>N$; we consider the problems

$$
\begin{aligned}
& \left\{\begin{array}{ccc}
\left\langle A^{p} u_{p}, v-u_{p}\right\rangle \geqslant\left\langle f, v-u_{p}\right\rangle & \text { a.e. in } \Omega \\
\forall v \in H_{0}^{1}(\Omega) & v(x) \leqslant \psi(x) & \text { a.e. in } \Omega \\
u_{p} \in H_{0}^{1}(\Omega) & u_{p}(x) \leqslant \psi(x)
\end{array}\right. \\
& \left\{\begin{array}{ccc}
\langle A u, v-u\rangle \geqslant\langle f, v-u\rangle & \text { a.e. in } \Omega \\
\forall v \in H_{0}^{1}(\Omega) & v(x) \leqslant \psi(x) & \text { a.e. in } \Omega \\
u \in H_{0}^{1}(\Omega) & u(x) \leqslant \psi(x) &
\end{array}\right.
\end{aligned}
$$

where $A$ and $A^{p}$ is as in Th. 5 .

Let be $u\left(u_{n}\right)$ the solution to the problem $(5.14),\left(\left(5.14_{\mathcal{2}}\right)\right)$ we have

$$
\left\|u_{p}-u\right\|_{\mathbb{C} \infty} \leqslant C p^{\bar{\alpha}_{12(N-2+3 \bar{x})}}
$$

where $C$ is a constant dependent as $\alpha, \beta$ and $\|p\|_{1, \beta}$ and $\bar{\alpha}$ is an Hölder exponent for $A^{p}, A$ and $W^{-1, p}$ in the De Giorgi-Nash theorem.

Let be now $u_{p}^{0}$ the solution to the problem

$$
A^{p} u_{p}^{0}=f
$$

and $u_{p}^{n}, n \geqslant 1$, the solution to the problem

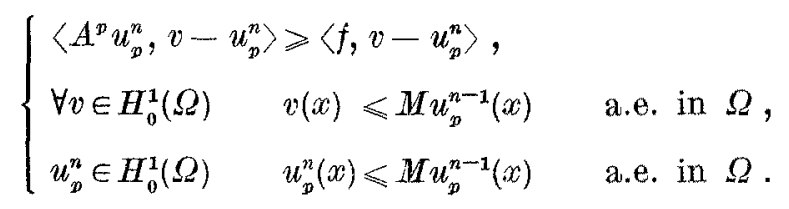


We show that

$$
\left\|u_{p}^{n}-u^{n}\right\|_{\mathfrak{C}^{\infty}} \leqslant C\left(n p^{-\bar{\alpha} / 2(N-2+\bar{\alpha})}+p^{-\overline{4}}\right) .
$$

From [7] we have (5.17) for $n=0$.

We suppose now (5.17) valid for $n-1$.

We have

$$
\left\|M u_{s}^{n-1}-M u^{n-1}\right\|_{\mathfrak{L}^{\infty}} \leqslant C\left((n-1) p^{-\bar{\alpha} / 2(N-2+\beta \bar{\alpha})}+p^{-\frac{1}{4}}\right) .
$$

Let be now $\bar{u}_{p}^{0}$ the solution to the problem

$$
\begin{cases}\left\langle A^{p} \bar{u}_{p}^{n}, v-\bar{u}_{p}^{n}\right\rangle \geqslant\left\langle f, v-\bar{u}_{p}^{n}\right\rangle, \\ \forall v \in H_{0}^{1}(\Omega) & v(x) \leqslant M u^{n-1}(x), \\ \bar{u}_{p}^{n} \in H_{0}^{1}(\Omega) & \bar{u}_{p}^{n}(x) \leqslant M u^{n-1}(x) .\end{cases}
$$

From the lemma $3,5,6$ we have

$$
\left\|\bar{u}_{p}^{n}-u^{n}\right\|_{\mathfrak{L}^{\infty}} \leqslant C^{\prime} p^{-\bar{\alpha} / 2(N-2+3 \bar{\alpha})}
$$

where $C^{\prime}$ depends only on $f, g$ and we can suppose $C>C^{\prime}$.

From Th. 1.4 [17] we have also

$$
\left\|u_{p}^{n}-\bar{u}_{p}^{n}\right\|_{\mathfrak{L}^{\infty}} \leqslant C\left((n-1) p^{-\bar{\alpha} / 2(N-2+3 \bar{\alpha})}+p^{\left.-\frac{1}{4}\right)}\right.
$$

then

$$
\left\|u_{p}^{n}-u^{n}\right\|_{\mathcal{L}^{\infty}} \leqslant O\left(n p^{-\bar{\alpha} / 2(N-2+3 \bar{\alpha})}+p^{-\frac{1}{4}}\right) .
$$

From the lemma 4 we have also

$$
\begin{aligned}
& \left\|u_{p}^{n}-u_{p}\right\|_{\mathbb{L}^{\infty}} \leqslant \frac{K}{n^{s}} \quad \forall s>0, n \geqslant \bar{n}_{s} \\
& \left\|u^{n}-u\right\|_{\mathfrak{C}^{\infty}} \leqslant \frac{K}{n^{s}}
\end{aligned}
$$

where $K$ is a constant independent on $p$.

From (5.22), (5.23) we have

$$
\left\|u_{p}-u\right\|_{\mathfrak{L}^{\infty}} \leqslant C\left(n p^{-\bar{\alpha} / 2(N-2+3 \alpha)}+p^{-\frac{1}{4}}\right)+K / n^{8} .
$$

Choosing $n^{s+1 / s}$ as the first integer $\leqslant p^{-\ddot{\alpha} / 4(N-2+3 \bar{\alpha})}$ we have

$$
\left\|u_{p}-u\right\|_{\mathfrak{L} \infty} \leqslant C p^{-\bar{\alpha} / 4(N-2+3 \bar{\alpha}) s /(s+1)} .
$$




\section{REFERENCES}

[1] A. Bensoussan - J. L. Lions, Nouvelle formulation des problèmes de contrôle impulsionnel, Comptes Rendus Acad. Sc. Paris, A 276 (1973), pp. 1189-1192.

[2] A. Bensoussax - J. L. Lions, Nouvelles méthodes en contrôle impulsionnel, Appl. Math. Opt., 1, (4) (1975), pp. 289-312.

[3] A. Bensoussan - J. L. Lions, Inéquations quasi-variationelles dépendantes d'une paramètre, Ann. Sc. Norm. Sup. Pisa.

[4] A. Bensoussan - M. Goursat - J. L. Lions, Contrôle impulsionnel et I.Q.V. stationnaires, Comptes Rendus Acad. Sc. Paris, A 276 (1973), pp. 1279-1281.

[5] A. Bensoussan - J. L. Lions - G. PaPanicolad, Sur quelques phenomènes asymptotiques stationnaires, Comptes Rendus Acad. Sc. Paris, A 281 (1975), pp. 89-94.

[6] M. Biroli, Sur la G-convergence pour des inéquations quasi-variationnelles, Boll. U.M.I., (5), 14-A (1977), pp. 540-551.

[7] M. BIROLI, An estimate on convergence for an homogeneisation problem for variational inequalities, in print Rend. Sem. Mat. Padova.

[8] M. BIRoLI, $G$-convergence for elliptic equations, variational inequalities and quasi-variational inequalities, in print Rend. Sem. Mat. Fis. Milano.

[9] E. De Giorgi - S. Spagrolo, Sulla convergenza degli integrali dell'energia per operatori ellittici del 2-ordine, Boll. U.M.I., (4) 8 (1973), pp. 391-411.

[10] J. L. JoLY - U. Mosco - G. Troraniello, Un résultat de régularité pour un'inéquation quasi-variationelle intervenant dans un problème de contrôle impulsionnel, Comptes Rendus Acad. Sc. Paris, A 279 (1974), pp. 937-941.

[11] Th. Laestoh, An uniqueness theorem for elliptic quasi-variational inequalities, J. Func. An., 18 (1975), pp. 286-287.

[12] J. L. Lrons, Sur quelques questions d'analyse, mécanique et contrôle optimal, Les presses de l'Université de Montréal, 1976.

[13] J. L. LIons, Asymptotic behavior of variational inequalities with highly oscillating coefficients, in Applications of Methods of Functional Analysis to Problems in Mechanics, Lecture Notes 503, Springer, 1976.

[14] J. L. MenaLd, Sur le problème de contrôle impulsionnel et l'inéquation quasi-variationelle degénerée associée, Comptes Rendus Acad. Sc. Paris.

[15] U. Mosco, Implicit variational problems and quasi-variational inequalities, in Nonlinear Operator and Calculul of Variations - Bruxelles 1975, Lecture Notes 543, Springer, 1976.

[16] F. Murat, Sur l'homogeneisation d'inéquations elliptiques du $2^{e}$ ordre ..., Lab. An. Num. Paris VI, no. 76013.

[17] J. P. Puel, Thèse, Lab. An. Num. Paris VI, 1975.

[18] G. Stampaconia, Problemi al contorno con soluzioni hölderiane, Ann. Mat. Pura Appl., 51 (1960), pp. 1-32.

[19] G. STampacchia, Equations elliptiques du second ordre à coefficients discontinues, Les presse de l'Université de Montréal, 1965.

[20] L. Tartar, Inéquations quasi-variationelles abstraites, Comptes Rendus Acad. Sc. Paris, A 278 (1974), pp. 1193-1196.

[21] L. Tartar, Cours Péccot, Collège de France, 1977.

[22] G. Troianiello, In print. 